\title{
Design of Quiet Rotorcraft Approach Trajectories: Verification Phase
}

\author{
Sharon L. Padula ${ }^{1}$ \\ NASA Langley Research Center, Hampton, VA, 23681
}

\begin{abstract}
Flight testing that is planned for October 2010 will provide an opportunity to evaluate rotorcraft trajectory optimization techniques. The flight test will involve a fully instrumented MD-902 helicopter, which will be flown over an array of microphones. In this work, the helicopter approach trajectory is optimized via a multiobjective genetic algorithm to improve community noise, passenger comfort, and pilot acceptance. Previously developed optimization strategies are modified to accommodate new helicopter data and to increase pilot acceptance. This paper describes the MD-902 trajectory optimization plus general optimization strategies and modifications that are needed to reduce the uncertainty in noise predictions. The constraints that are imposed by the flight test conditions and characteristics of the MD-902 helicopter limit the testing possibilities. However, the insights that will be gained through this research will prove highly valuable.
\end{abstract}

\section{Nomenclature}

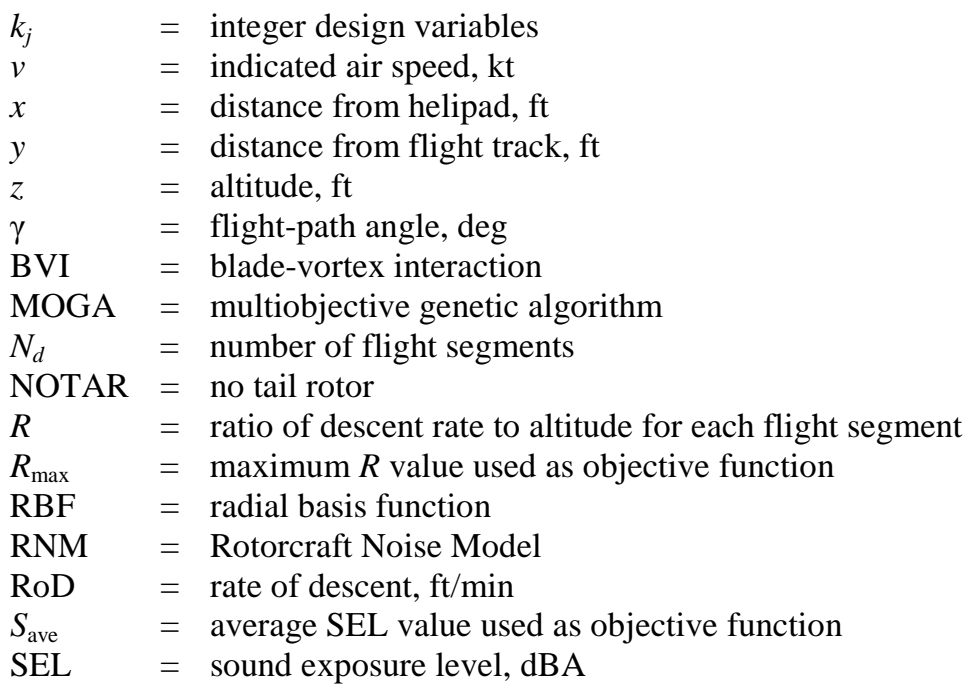

\section{Introduction}

$\mathrm{T}$ The U.S. Government military and civilian laboratories perform fundamental research in noise prediction methods and noise reduction technologies, and sponsor rotorcraft flight and wind-tunnel testing (e.g., see Refs. 1-3). This fundamental research has produced a greater understanding of rotorcraft noise and has led to the development of a number of valuable software tools, such as the Rotorcraft Noise Model (RNM). ${ }^{4}$

The Subsonic Rotary Wing (SRW) project under the NASA Fundamental Aeronautics Program seeks to develop multidisciplinary design optimization tools that enable the design and operation of safe and environmentally compatible rotorcraft. One segment of the SRW project emphasizes vehicle acoustics and noise propagation tools. For example, the present study uses the RNM software to create trajectory optimization techniques for reducing community noise near helipads.

\footnotetext{
${ }^{1}$ Senior Research Scientist, Mail Stop 442, AIAA Assoc. Fellow.
} 
The initial phase of this research was completed in July of 2009 and reported in Ref. 5. A multiobjective genetic algorithm (MOGA) with 14 design variables was used to successfully produce helicopter approach trajectories that minimized community noise and avoided abrupt decelerations and high rates of descent (RoD) that would be unacceptable to pilots and passengers. The optimized trajectories were significantly quieter than a standard 6-deg approach.

This paper briefly describes the trajectory optimization problem, provides sample results, and makes recommendations for flight testing and validation of the results. More complete details about the noise prediction software, optimization problem, design variables, objective functions, and constraints are available in Refs. 4 and 5. The present paper discusses issues that are associated with the RNM software, additional constraints, and the database of noise hemispheres. The paper extends the methods that are introduced in Ref. 5 and discusses the application of these methods to the plans for an October 2010 MD-902 flight test.

\section{Quiet Rotorcraft Approach Trajectories}

\section{A. RNM Methodology}

The RNM code is a simulation program that models sound propagation and predicts noise levels at microphone locations on the ground. The code includes subroutines to model the changes in noise level caused by spherical spreading, weather conditions, a layered atmosphere, and ground effects. However, the code does not model flight dynamics; a helicopter trajectory is specified by a set of $(x, y, z)$ way points in space and by speed $v$ and flight-path angle $\gamma$ at each way point. Constant acceleration (or deceleration) is assumed between way points.

The RNM code uses a database of measured or predicted noise hemispheres as input to predict the noise footprint for a given rotorcraft trajectory (Fig. 1). Each noise hemisphere tabulates noise as a function of frequency and spherical angle at a fixed distance from the craft. The database of noise hemispheres must cover a range of possible helicopter speeds, rates of descent, and approach path angles.

The basic elements of the helicopter trajectory analysis and optimization are pictured in Fig. 1. The approach trajectory is described by a set of $N_{\mathrm{d}}$ straight flight-path segments with linearly varying parameters such as speed and altitude. The RNM code estimates the helicopter position as a function of time and propagates the noise from each helicopter position to a number of microphone locations on the ground. Typical RNM outputs are an A-weighted sound exposure level (SEL) for a single microphone or a color contour plot (i.e., an SEL noise footprint) for an array of

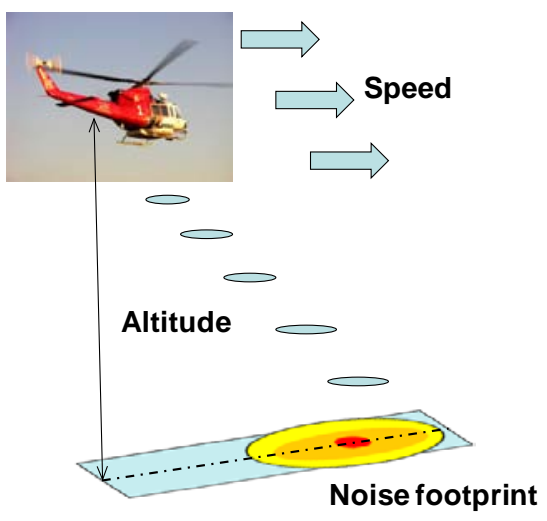

Figure 1. Flight path design variables. microphones.

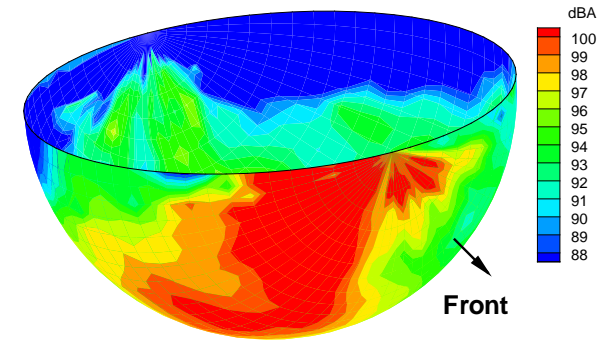

(a) Typical MD-902 hemisphere.

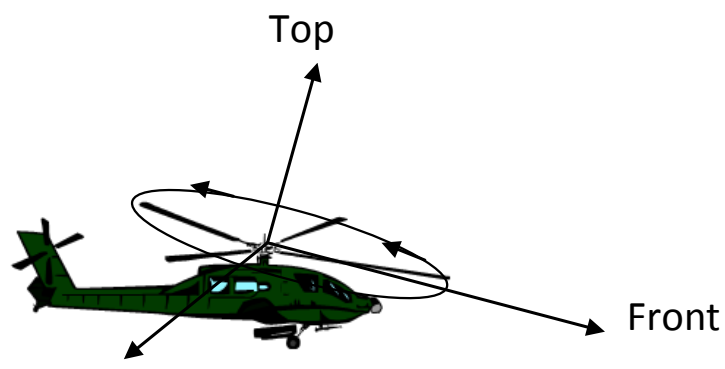

Advancing Side

(b) Hemisphere origin at rotor hub.

Figure 2. Source noise hemispheres. 
The predicted noise level for any microphone location depends on the characteristics of the helicopter source noise as well as on the approach trajectory. The source noise at a fixed radius from the

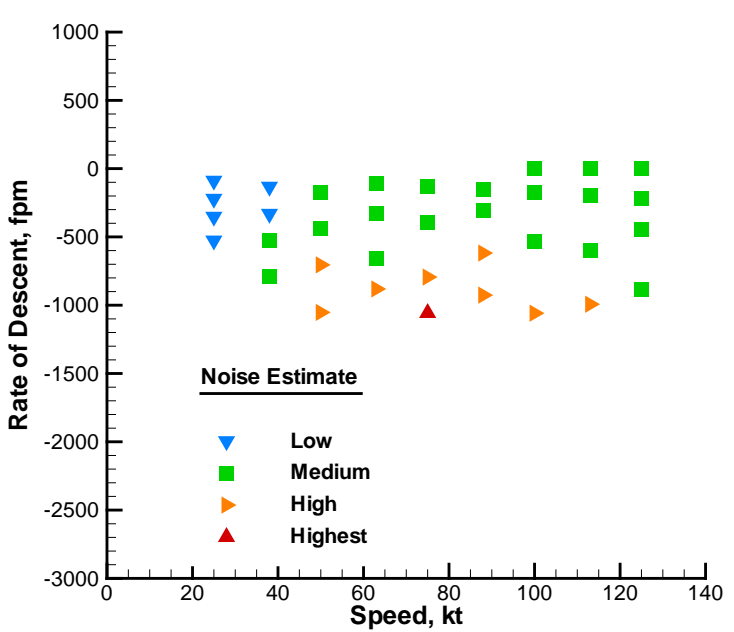

Figure 3. Predicted source noise hemispheres.

certain descent conditions the vortices that are shed from one rotor blade come in contact with the next rotor blade and create exceptionally high noise levels called blade-vortex interaction (BVI) noise.

Figure 3 indicates the relative noise levels for 36 different hemispheres that are used to predict source noise for the MD-902 helicopter. This figure was created by inspecting a color contour plot of each hemisphere and estimating the average noise level on the advancing side. Figure 3 suggests that a forward speed of 80 knots and a descent rate of $-1,000 \mathrm{ft} / \mathrm{min}$ will produce the highest source noise. Based on this figure, one would not expect the optimized approach trajectory to include any rates of descent near $-1,000$ $\mathrm{ft} / \mathrm{min}$ or any speeds near 80 knots.

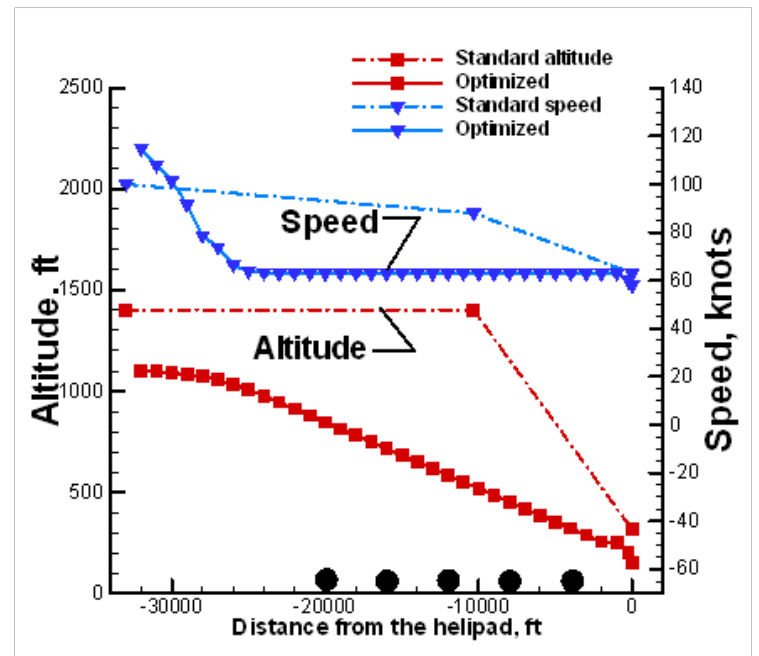

Figure 4. Initial optimization results compared to standard approach trajectory.

\section{B. Initial Optimization Results}

Reference 5 concluded that selecting appropriate design variables to parameterize the approach path was the key to a successful optimization. In that reference, 14 integer design variables produce a wide variety of candidate approach paths. The design variables select the initial altitude, the initial speed, and the percentage of change in speed and descent rate for each of the flight-path segments. The values of the integer design variables are converted into the physical distances, altitudes, and speeds by using simple arithmetic equations which are given below. These physical descriptions of the approach path become the way points used by the RNM code to predict noise.

The initial optimization results that were reported in Ref. 5 are reproduced in Fig. 4. The black dots indicate five microphone locations at which the SEL is minimized. The dashed line represents a standard approach trajectory that is based on a trajectory that was tested in Ref. 1 . The solid line represents the optimized trajectory.

It is interesting to compare the trajectories shown in Fig. 4. The standard trajectory gradually decelerates to 60 knots and has a flight-path angle of 6 deg. Notice that the optimized speed drops quickly to 60 knots and that the flight-path angle is much less than 6 deg. The optimized trajectory is made up of 32 flight-path segments, but it appears to be a smooth and comfortable descent. Fig. 5 compares the noise footprints for the standard and optimized trajectories. Again, the black dots indicate locations where noise is minimized. The noise footprint of the optimized approach trajectory is significantly quieter than the footprint of the standard approach trajectory. 


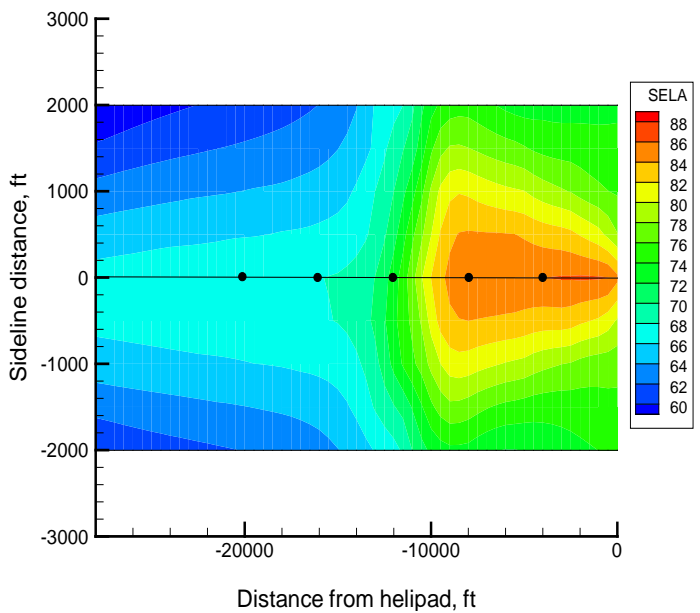

(a) Standard approach path.

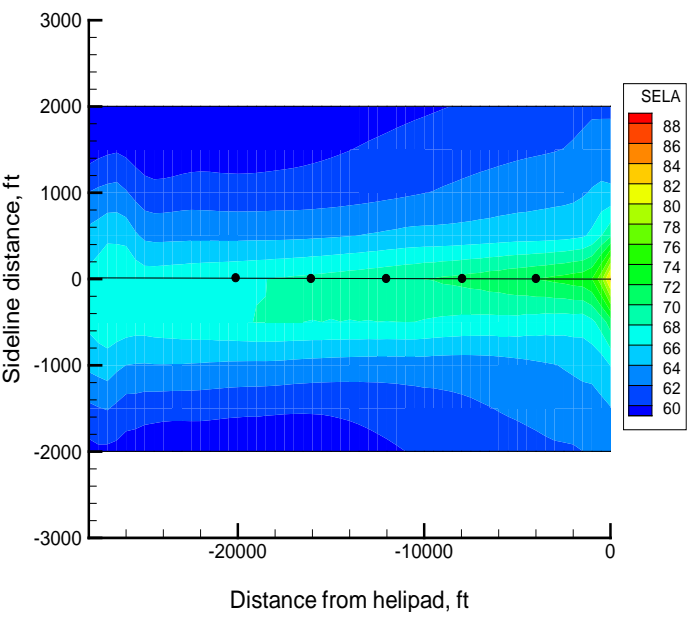

(b) Optimized approach path.

Figure 5. Initial optimization noise footprints.

\section{Flight Test Preparations}

The NASA Langley Research Center, the U.S. Army's Joint Research Program Office, and the University of Maryland plan to conduct the Maneuver Acoustic Flight Test at Eglin Air Force Base, FL in October 2010. The primary objective of the flight test is to characterize the noise that is created by a maneuvering helicopter. A secondary objective is to augment the existing source-noise database that is used by both the RNM code and by military acoustic detection prediction codes. The same microphone array and helicopter instrumentation that is required for these objectives is suitable for testing optimized approach trajectories. Thus, an important SRW goal of developing low-noise approach procedures can be addressed by this flight test without a significant impact to cost or schedule. However, any optimized flight trajectory must be deemed safe to fly and informative to test or it will not be added to the flight test matrix.

The test vehicle is a specially equipped MD-902 helicopter similar to the vehicle shown in Fig. 6. The MD-902 is a five-bladed helicopter with a maximum takeoff gross weight of 6,250 lb and maximum speed of 140 knots. The helicopter has a main rotor with a diameter of $33.84 \mathrm{ft}$ and a NOTAR fan in place of a conventional tail rotor.

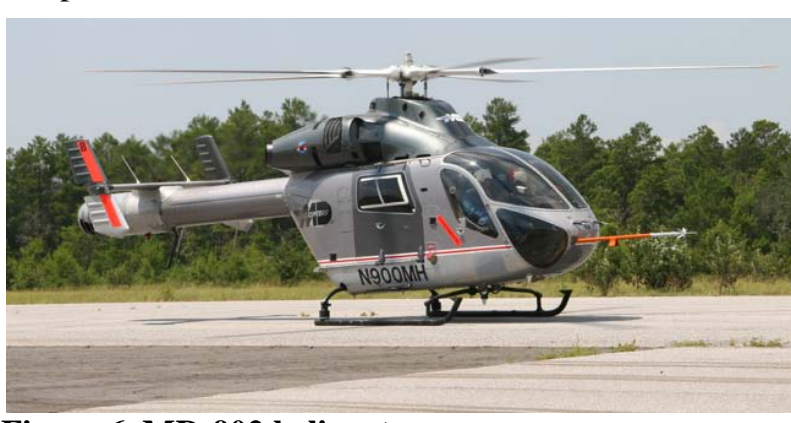

Figure 6. MD-902 helicopter.

The seven-day flight-test program promises to acquire high-quality acoustic data as a result of the skilled personnel, favorable environment, and extensive instrumentation to be used for this test. For example, the NASA Langley Research Center's differential global-positioning-system (GPS) tracking and guidance system will acquire vehicle position data with centimeter accuracy. The flight-test engineer and the test pilot, both from Boeing's Rotorcraft Systems facility in Mesa, AZ, will compare real-time position information against desired vehicle position to fly the specified trajectories. The vehicle will be instrumented so that flight conditions, pilot inputs, and tip path plane angle can be recorded along with the high quality acoustic measurements.

\section{A. Available Source Noise Hemispheres}

The initial optimization results that are reported in Ref. 5 were based on predicted source-noise hemispheres (see Fig.3). These 36 hemispheres were generated as explained in Ref. 3. These predictions would be expected to match 
wind-tunnel measurements for an isolated rotor under carefully controlled tunnel conditions; however, the predicted source-noise hemispheres do not match the total vehicle noise under expected flight-test conditions. ${ }^{3}$ Thus, the first step in preparation for the flight test is obtaining measured source-noise hemispheres for the MD-902.

The available set of measured MD-902 hemispheres covers an adequate range of speeds and descent rates (see Fig. 7), but the number of available hemispheres is lower than the 36 hemispheres that were used in Ref. 5. Moreover, the measured data are not uniformly distributed over each hemisphere but rather show a great deal of data near the bottom of the hemisphere and no data in some spherical directions. Ref. 2 contains a full description of the measurement process, and Ref. 6 contains a discussion of the possible shortcomings of the resulting database of noise hemispheres.

The partial MD-902 hemispheres are only a minor annoyance to researchers who are predicting noise for simple flight paths (e.g., those with a constant speed and flight-path angle). However, these partial hemispheres cause major problems for optimization procedures that process hundreds of unique flight paths such as those shown in Fig. 4. Initial trials with the available MD-902 hemispheres showed that the optimization process exploited the lack of data and converged to trajectories that were clearly suboptimal.

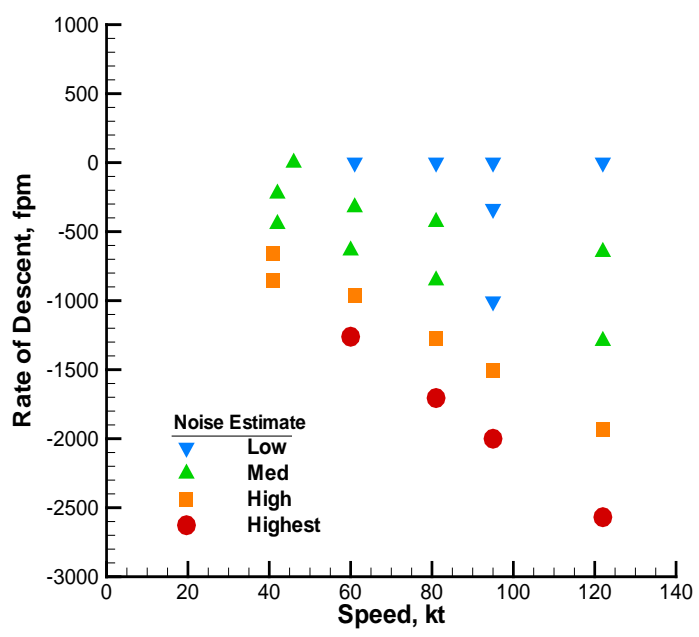

The solution to this dilemma is to improve the existing hemispheres through the use of interpolation as described and verified in Ref. 6. Several interpolation methods are available, but a radial basis function (RBF) interpolation provides a significantly more accurate representation of the radiated noise. ${ }^{6}$ A major strength of RBF interpolation is that the accuracy of the method is relatively insensitive to the geometry of the problem; consequently, RBF interpolation is well suited to the interpolation of scattered data on complex surfaces. The new interpolation scheme applies smooth and nonoscillatory basis functions across the hemisphere to estimate noise levels on the regular grid of spherical angles required by RNM. ${ }^{6}$ Areas near the bottom of the hemisphere with too much measured data are averaged to provide a coarser distribution of data points. Areas of the sphere with no data are smoothed by appropriate manipulation of the boundary conditions. A known strength of the RBF method is that it guarantees that the interpolation results will exactly match the data at all control points. The major drawback to RBF methods is the computational time that is required to fit the data. Faster computers and efficient and stable solution methods have partially overcome this drawback. Ref. 6 introduces both a preconditioning scheme and a local/global solver that make the computations faster and more stable.

The RBF method is used to process all of the available MD-902 data into smooth and accurate hemispheres. Given a set of fully populated hemispheres, a second interpolation creates new hemispheres to fill in some of the gaps in the speed and descent ranges. Figure 7 indicates the relative noise levels of the final set of hemispheres. Both Fig.7 and Fig. 3 show relative noise levels for the MD-902 as a function of speed and RoD, but Fig. 3 is based on predicted hemispheres for an isolated main rotor while Fig. 7 is based on measured data for the full vehicle.

\section{B. Constraints}

The initial optimization formulation must be modified to include constraints that address pilot safety and pilot workload. The initial optimization formulation used a single constraint to limit the maximum rate of descent. Ref. 5 defines a variable $R$ that is the ratio of the RoD divided by the altitude. The value of $R$ is determined for each of the 32 flight-path segments and the maximum value $R_{\max }$ is constrained. One of the conclusions established in Ref.5 is that the convergence of the MOGA to an optimized flight path is sensitive to the value of this constraint. 


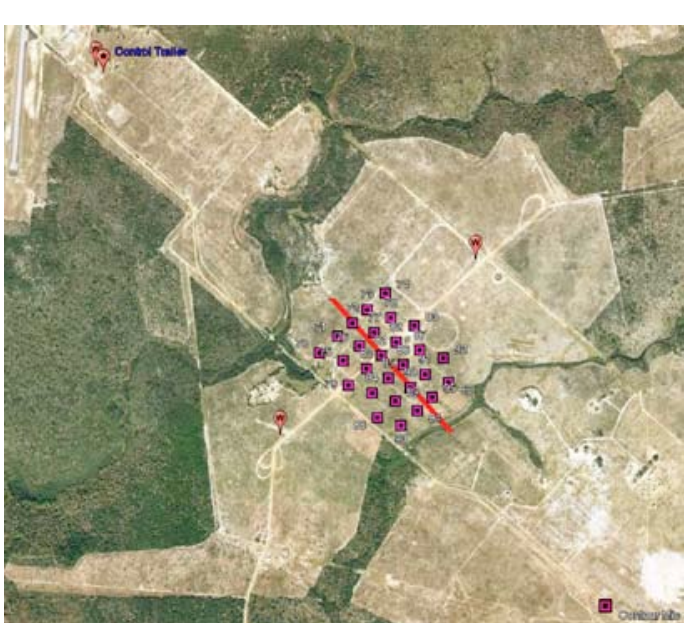

(a) Satellite view of Eglin AFB.

\section{Figure. 8 Proposed microphone locations}

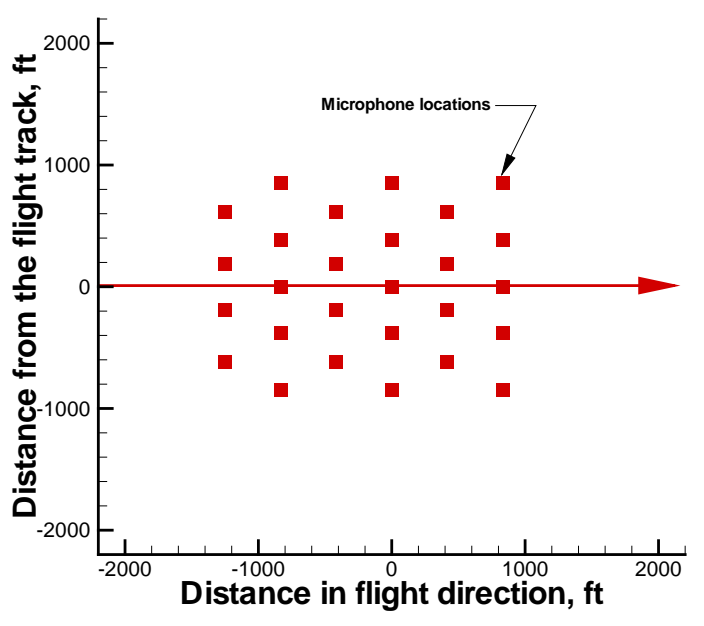

(b) Array of 27 microphones.

Conversations with engineers and helicopter pilots suggested that the initial optimization results did not represent acceptable flight paths. These professionals suggested several additional constraints on the optimization formulation. First, a minimum speed of 80 knots and a minimum flight-path angle of 3 deg are preferred when flying the MD-902 below 1,000 ft in altitude. These minimums provide a safer flight and one that is more acceptable for the pilots and passengers. Second, the pilot's workload is lower if changes in speed and changes in altitude do not occur simultaneously. Finally, any abrupt acceleration (or deceleration) that exceeds $0.1 \mathrm{~g}$ is uncomfortable for passengers and may cause a variation in the source noise that cannot be completely accounted for by the RNM software (e.g., see Ref. 6 for a discussion of acceleration effects).

A look at the test plan and the requirements document for the Maneuver Acoustic Flight Test revealed additional issues. The original noise footprints (see Fig. 5) extended 33,000 ft in the flight direction and 3,000 ft to each side of the flight track. The optimization procedure reduces noise in this large area to decrease the likelihood that the rotorcraft operations will disturb the surrounding community. However, the test plan prescribes an array of 27 microphones within a much smaller $\left(1,000 \mathrm{ft}^{2}\right)$ area shown in Fig. 8 . An expanded microphone array is impractical; microphones must be placed on flat and unobstructed terrain and the noise produced by the helicopter must be much louder than the noise from other traffic on the Eglin AFB. The concern is that this microphone array may not be sufficient to test the effectiveness of the optimization procedure.

\section{Optimization Problem Formulation}

The optimization problem that is described in Ref. 5 is summarized here. The candidate approach paths are defined by the 14 integer design variables $k_{\mathrm{j}}$. The first 11 design variables determine the speed $v$ and the altitude $z$ at the end of the first 11 flight-path segments, whereas $k_{12}$ determines the index of the discrete glide slope. Finally, $k_{13}$ and $k_{14}$ adjust the initial speed $v_{0}$ and altitude $z_{0}$ :

$$
\begin{array}{ll}
v_{j}=v_{j-1}\left(1.0-0.02 k_{j}\right) & 1 \leq k_{j} \leq 9, j=1, \ldots, 11 \\
v_{0}=130-10 k_{13} & 1 \leq k_{13} \leq 5 \\
z_{0}=100 k_{14} & 10 \leq k_{14} \leq 15 \\
x_{1}=-32000\left(1-k_{15} / 12\right) & 0 \leq k_{15} \leq 8
\end{array}
$$

For any path segment, the reduced speed $v_{\mathrm{j}}$ is determined from the speed in the previous segment by using Eq. (1). For example, if $k_{\mathrm{j}}$ is equal to 2, then the new speed is 96 percent of the previous speed. Given the speed $v_{\mathrm{j}}$ and the index $k_{12}$, the appropriate RoD can be estimated. Given the RoD and the current altitude $z_{\mathrm{j}-1}$, the next altitude $z_{\mathrm{j}}$ can be calculated. The calculated values of speed and altitude are used until the terminal state is reached; the remaining flight-path segments have a fixed $v$ and $z$. The total number of flight-path segments is fixed at $N_{\mathrm{d}}=32$. This procedure creates smoothly varying descent flight segments with monotonically decreasing speed and altitude. 
However, for some values of the design variables, the final values of $v$ and $z$ will be higher than the desired terminal state. All such trajectories must be disallowed by the optimization procedure.

In preparation for flight testing, the optimization problem that is described in Ref. 5 was modified. The reformulation of the problem uses the same integer design variables but makes the following changes. All flight paths start at $x=-33,000 \mathrm{ft}, z=z_{0}$ and $v=v_{0}$; all paths have identical terminal states, namely $x=0$

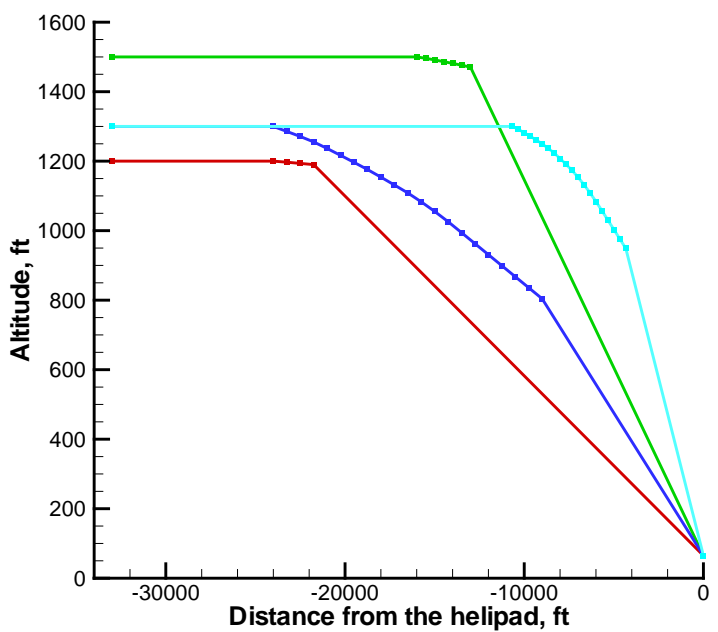

Figure 9. Typical approach trajectories generated by varying design variables $N_{\mathrm{d}}$ and $\boldsymbol{k}_{\mathrm{j}}$.

$\mathrm{ft}, z=65 \mathrm{ft}$ and $v=80$ knots. The number of flight path segments varies between 6 and 24 and is controlled by a new design variable $N_{d}$. Finally, the start of descent distance $x_{1}$ is controlled by a new design variable $k_{15}$. As a consequence of the reformulation, for the typical generated approach trajectory speed decreases during the initial flight path segments, and the speed and the angle are constant during the final flight-path segment. The length of the first and the last segment is controlled by the MOGA via the new design variables. Figure 9 illustrates some of the possible trajectories that can be generated with the modified MOGA.

The reformulated problem places some additional constraints on the flight-path angle $\gamma$ and on the acceleration $\partial v / \partial t$. All flight-path segments are examined in post-processing; the minimum and maximum values of $\gamma$ and $\partial v / \partial t$ are calculated. The constraint is marked as feasible if -12 deg $<\gamma<-3$ deg for all flight-path segments below $1,000 \mathrm{ft}$ in altitude. A second constraint is marked as feasible if $-0.1 \mathrm{~g}<\partial v / \partial t<0.0 \mathrm{~g}$ for all flight-path segments. The optimization problem can be stated as:

$$
\begin{array}{ll}
\text { Choose } & N_{\mathrm{d}} \text { and } k_{\mathrm{j}} \\
\text { Minimize } & \left(R_{\max }-1\right)^{2}+S_{\text {ave }} \\
\text { Subject to } & -12<\gamma<-3 \\
& -0.1<\partial v / \partial t<0.0
\end{array}
$$

where $S_{\text {ave }}$ is a measure of the noise at the microphone locations and $R_{\max }$ is a measure of the maximum RoD over all flight segments.

\section{Uncertainty of Noise Predictions}

As an initial test of the new optimization formulation, two approach trajectories were produced. Both trajectories minimized $\left(R_{\max }-1\right)^{2}$ and met all of the constraints; however, one trajectory minimized $S_{\text {ave }}$ and the other maximized $S_{\text {ave }}$. As expected, the difference in the predicted footprints for the two trajectories was significant. However, the plots of speed and altitude as a function of time were quite similar. This indicated an error in the RNM predictions because nearly identical flight-path segments should produce nearly identical noise footprints.

The error occurs after the RNM code determined the speed and angle of the current flight-path segment and before it propagated the noise from that segment to the ground. Given a speed and angle, the RNM code must choose a source-noise hemisphere. RNM version 7.2.2 is configured to use the nearest source-noise hemisphere rather than to interpolate between several available hemispheres. Refer to Fig. 7 and consider a case for which the current speed is 100 knots and the descent rate is $-1,300 \mathrm{ft} / \mathrm{min}$. If the speed is increased to $102 \mathrm{knots}$, then RNM uses the 120-knot hemisphere and predicts a lower noise value, while if the speed is decreased to 98 knots, then RNM uses the 80-knot hemisphere and predicts a higher noise value. In this way, two nearly identical approach trajectories can be constructed to have very different $S_{\text {ave }}$ values predicted by the RNM code. This is not really an error in RNM, so much as a lack of source-noise data for flight conditions in which the source noise is very sensitive 
to both RoD and speed. The lack of data is partly overcome through the use of the RBF interpolation; Fig. 7 represents the resulting set of source-noise hemispheres.

This situation was encountered and overcome in Ref. 5. The solution is to run two versions of RNM: one that encourages interpolation between hemispheres and one that does not. The difference between the two $S_{\text {ave }}$ predictions can be used as an added constraint in the optimization process. In this way, regions of the design space where the noise prediction is uncertain are penalized and the optimizer is able to converge on acceptable solutions.

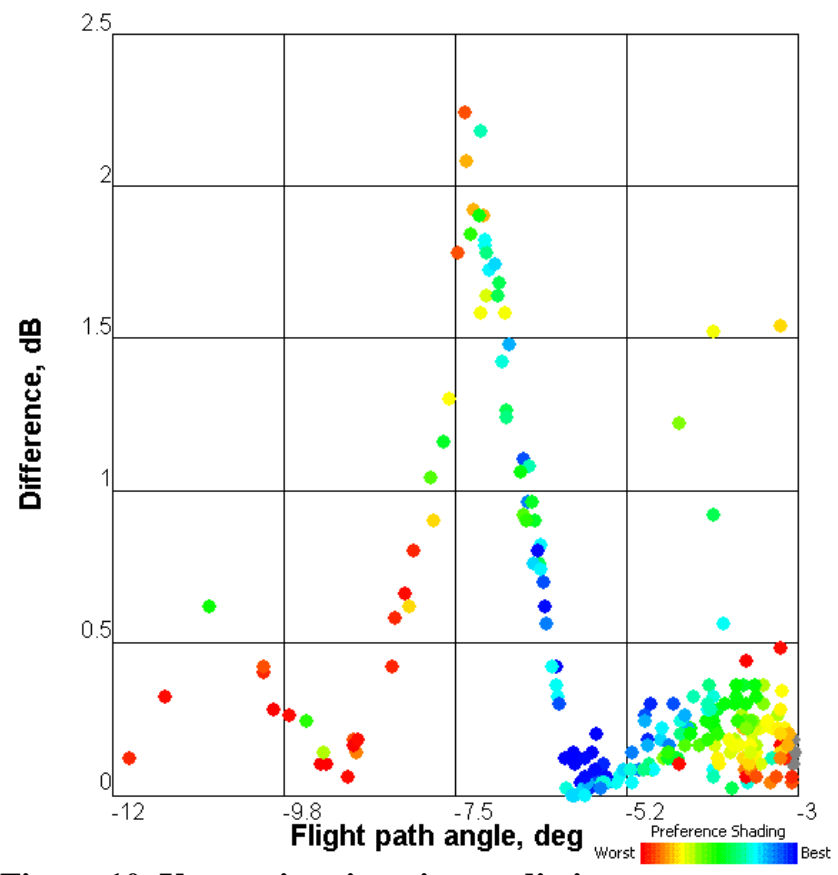

Figure 10. Uncertainty in noise prediction.

The magnitude of the uncertainty can be seen in Fig. 10. Here a Latin hypercube design of experiments (DOE) is used to produce 400 random approach trajectories that are similar to those shown in Fig. 9. Both versions of the RNM code process each trajectory and the difference in $S_{\text {ave }}$ is plotted against the maximum flight-path angle. The value of the objective function (see Eq. 2) for each trajectory is indicated by the color of the dots in Fig. 10 . Blue dots indicate the best values of $S_{\text {ave }}$ and $R_{\max }$, while red dots indicate the worst values. Clearly, the trajectories that have flight-path angles near -6 degrees have good values of the objective function and low uncertainty in predictions; trajectories with angles near -7.5 degrees have the highest uncertainty in predictions. Close examination of the DOE results supports this impression and indicates that the maximum $\gamma$ usually occurs during the final flight-path segment and has a large influence on the objective function value.

The results seen in Fig. 10 are quite surprising. The most likely explanation for high noise and high uncertainty for flight-path angles

near -7.5 degrees is that the helicopter experiences BVI for some of these flight conditions. This is surprising because most helicopters experience BVI at lower flight-path angles. It is known that helicopter source noise increases dramatically when BVI is encountered. Unfortunately, the MD-902 data set contains no source noise measurements for flight-path angles between -6 deg and -9 deg. Therefore, the exact flight conditions that cause BVI are hard to predict. Measuring source noise data for flight-path angles near -7.5 deg at several air speeds will be a key objective for the October 2010 flight test.

\section{Results}

The optimization problem that is described in Eq. 2 is repeated with variations on the objectives and the constraints. In one variation, the locations of the microphones that are used to predict $S_{\text {ave }}$ are changed from the five locations shown in Fig. 5 to the three locations suggested by the Federal Aviation Administration (FAA) noise certification test for helicopters. In a second variation, the definition of $S_{\text {ave }}$ is changed. For example, $S_{\text {ave }}$ can be defined as an average of the five predicted SEL values or an average of the difference between the current and the standard SEL values. Finally, $S_{\text {ave }}$ can represent an average of all 549 locations that are used to produce noise footprints. All of these variations are discussed in Ref. 5. The major effect of these changes is to change the weighting between the two objectives.

The MOGA is run with variations in the constraints as well as variations in the objective function. The constraints given in Eq. 2 limit the flight-path angle and the acceleration. An additional constraint can be placed on the difference between the two RNM predictions; a difference of $0.5 \mathrm{~dB}$ is considered acceptable. Finally, the side constraints on the design variables can be adjusted from the values that are given in Eq. 2. For example, $1 \leq k_{\mathrm{j}}$ produces flight-path segments in which the helicopter decelerates until the terminal conditions are met. Allowing the bounds on $k_{\mathrm{j}}$ to include zero permits level flight segments with a constant speed.

The results are consistent for all variations of the objective and the constraints. The optimal flight paths are similar to the 6-deg standard approach. The standard flight path makes the least noise and has acceptable 
acceleration and RoD values. The MOGA can converge to a 3-deg approach but only if the rate of descent objective is emphasized over the noise objective.

Figure 11 illustrates the trade-off between

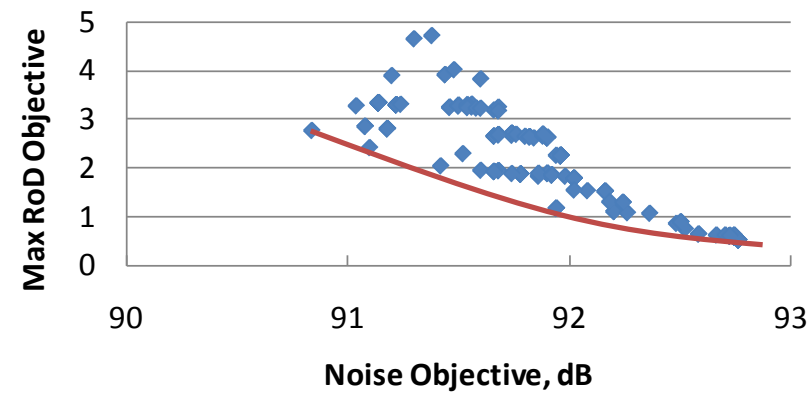

Figure 11. Plot of 400 solutions generated by MOGA (red line indicates pareto boundary). the two objectives. This figure is a scatter plot of the objective-function values for approximately 400 flight paths that were generated by the MOGA. Notice that, for this set of objectives and constraints, the first objective $S_{\text {ave }}$ varies between 90 and $93 \mathrm{~dB}$ while the second objective $\left(1-R_{\max }\right)^{2}$ varies between 0.5 and 5.0. The red line indicates the pareto optimal boundary. The MOGA will converge to one end of this curve or the other depending on the weighting of the two objective functions.

Figure 12 shows the 6-deg and 3-deg approach trajectories that are recommended for flight testing. Both of these trajectories were discussed with test engineers and with an MD-902 pilot. With minor adjustments near the transition between level flight and descending flight, both trajectories were deemed safe to fly. Of the two trajectories, the 6-deg trajectory will have a higher pilot workload because the speed and altitude decrease simultaneously. Moreover, both trajectories transition from level flight at an altitude above 1,000 ft and end at an altitude of $65 \mathrm{ft}$. During the flight test, the pilot might transition more gradually and might pull out of the descent at a higher altitude. These changes in approach trajectory will modify the noise footprint near $x=0 \mathrm{ft}$ and $y=0 \mathrm{ft}$ but should have little effect elsewhere.

Figure 13 compares the noise footprints for the recommended flight paths. Unfortunately, the noise difference between the two paths is relatively minor. Furthermore, the difference between the two paths will be hard to capture given the available microphone array (see the dashed box in Fig. 13).

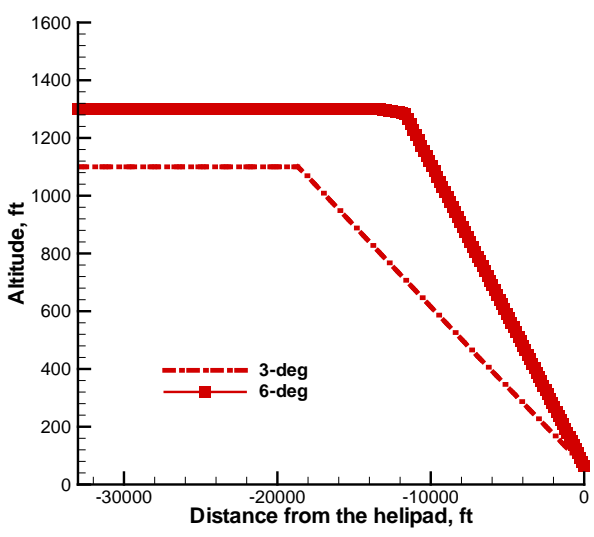

(a) Altitude profile.

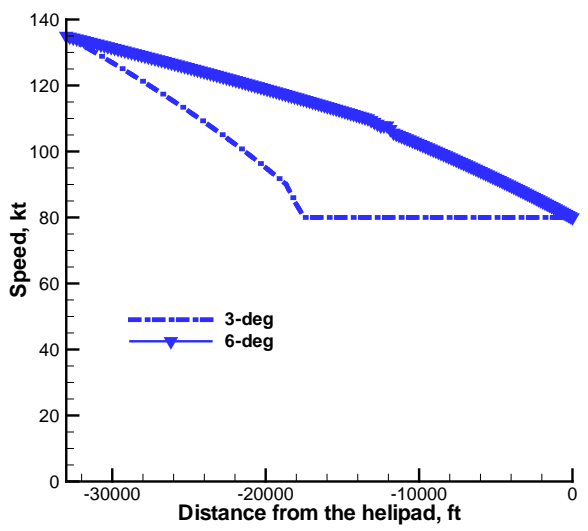

(b) Speed profile.

Figure 12. Flight paths recommended for testing. 


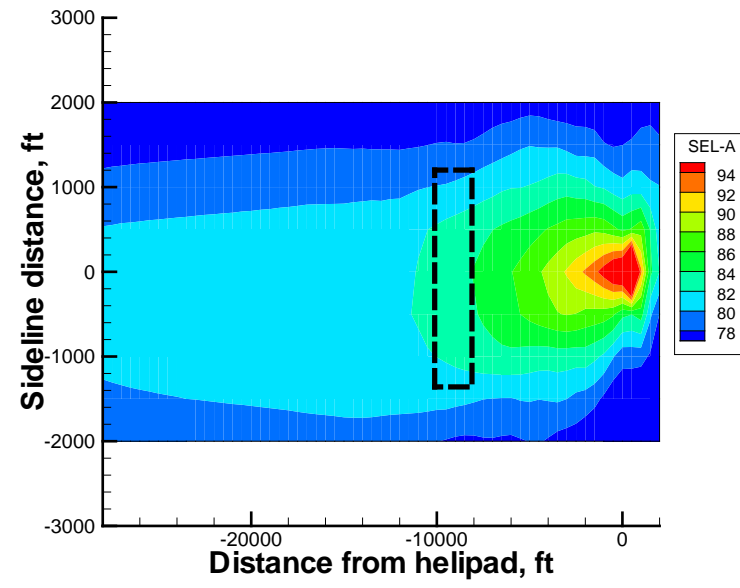

(a) Noise footprint for 6-deg path.

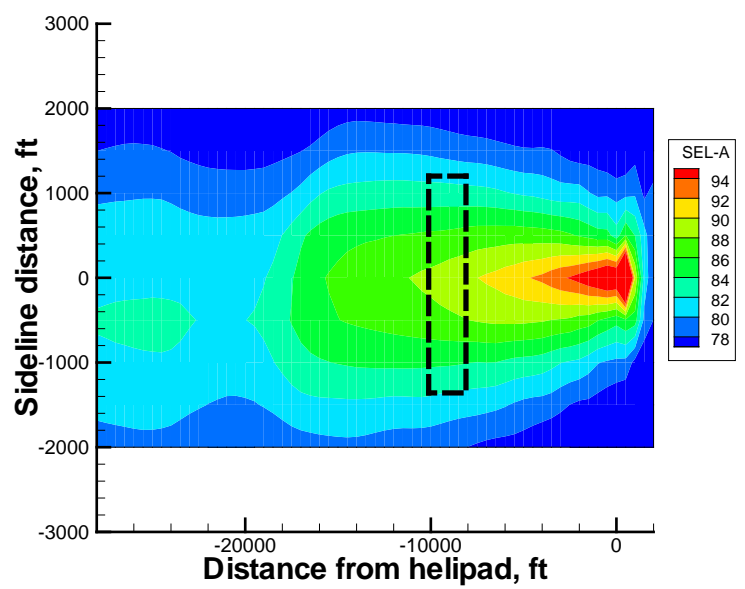

(b) Noise footprint for 3-deg path.

Figure 13. Optimization results; dashed box indicates approximate size of microphone array.

The results of this optimization study are somewhat disappointing. Obviously, the team had hoped for an optimized flight trajectory that was much quieter than the standard 6-deg trajectory that is used for noise certification. Moreover, the team had hoped that the difference between the optimized trajectory and the standard trajectory would be confirmed by flight testing.

These hopes for an improved approach trajectory were not realized for a number of reasons. First, the design space is quite limited. The minimum speed and minimum flight-path angle are more restrictive than those that were used in Ref. 5. For example, Fig. 14 identifies the subset of all measured source-noise hemispheres that meet the speed and angle constraints. Adding a constraint to account for the uncertainty in the RNM predictions shrinks the design space further as does the constraint on maximum acceleration. Second, the measured source noise hemispheres may not adequately model the helicopter that will be used in the flight test. The available data does not cover all of the flight conditions in the design space as well as the team had hoped. The RBF interpolation is helpful but cannot provide all the required source noise data. The lack of data between -6 deg and -9 deg is especially troublesome. Furthermore, the test vehicle will be modified to add trim tabs to each rotor blade. These tabs will enable accurate measurements of the tip path plane orientation, but they could change the noise that is produced by the main rotor. The above factors added to project priorities and budget constraints make it likely that the test of the optimized trajectories will be given a low priority on the test matrix.

\section{Conclusion}

Physical testing is an excellent opportunity to improve any mathematical analysis or optimization strategy. The 2010 Maneuver Acoustic Flight Test at Eglin Air Force Base provides a means of verifying and improving the noise propagation and approach trajectory optimization software tools.

Working with the maneuver test team has already proved beneficial. The trajectory optimization software has been improved with the addition of realistic constraints and the incorporation of pilot feedback. The strengths and 
weaknesses of the measured source-noise data for the MD-902 helicopter have been evaluated and the usefulness of this data has been improved. In addition, the Rotorcraft Noise Model (RNM) code has been thoroughly exercised and several improvements to the code are available in version 7 of that software.

Adding trajectory optimization to a test matrix presents problems and opportunities to the maneuver test team. As with any test program, if new potential flights are proposed then the prioritization of all flights becomes more difficult. The flights to verify optimized trajectories and to improve the accuracy of RNM predictions remain a secondary priority in these tests. The present plan is to allocate one day of the seven day flight test schedule to collect this data. This one day of testing will measure the noise footprints for several 6-deg flight paths with decreasing speed profiles. These flight paths are similar to the best trajectory identified by the optimization process. Other flights will address the uncertainty of RNM predictions as a function of flight-path angle. For example, based on the need for additional data near $\gamma=-7.5$ deg, several flights have been added to the test matrix.

In conclusion, the Maneuver Acoustic Flight Test in October 2010 will improve our ability to predict and optimize helicopter approach noise. New source noise data will decrease the uncertainty in the noise footprint predictions. Post-flight discussions with the pilot and comparison between the desired vehicle position and the actual vehicle position will improve the trajectory models. Test results from the 6-deg flight paths with decreasing speed profiles will suggest better ways to formulate approach trajectory optimization problems. Thus, even though many factors have limited the testing possibilities, the insights that will be gained through this research will prove highly valuable.

\section{Acknowledgments}

The author wishes to thank Eric Greenwood of NASA Langley Research Center for providing smoothed MD902 source noise hemispheres and for reviewing and improving this manuscript. She acknowledges William A. Decker, James E. Lindsey, and Robert A. Morris of NASA Ames Research Center for lively discussions of trajectory optimization techniques and helicopter flight constraints. She thanks Elif Kurklu of Stinger Ghaffarian Technologies, Inc. for sharing the results of alternate trajectory optimization techniques.

\section{References}

${ }^{1}$ Chen, R. N., Hindson, W. S., and Mueller, A. W., “Acoustic Flight Tests of Rotorcraft Noise-Abatement Approaches Using Local Differential GPS Guidance,” NASA TM-110370, September 1995.

${ }^{2}$ Conner, D. A., Burley, C. L., and Smith, C. D., "Flight Acoustic Testing and Data Acquisition for the Rotor Noise Model (RNM),” Presented at American Helicopter Society 62nd Annual Forum, Phoenix, AZ, May 9-11, 2006.

${ }^{3}$ Boyd, D. Douglas, "Initial Aerodynamic and Acoustic Study of an Active Twist Rotor using a Loosely Coupled CFD/CSD Method," Presented at the $35^{\text {th }}$ European Rotorcraft Forum, Hamburg, Germany, September 22-25, 2009.

${ }^{4}$ Conner, D. A., and Page, J. A., “A Tool for Low Noise Procedures Design and Community Noise Impact Assessment: The Rotorcraft Noise Model (RNM),” Presented at Heli Japan 2002, Tochigi, Japan, November 11-13, 2002.

${ }^{5}$ Padula, S. L., Burley, C. L., Boyd, D. D., and Marcolini, M. A., “Design of Quiet Rotorcraft Approach Trajectories,” NASA TM-2009-215771, June 2009.

${ }^{6}$ Greenwood, Eric, “A Physics-Based Approach to Characterizing Helicopter External Noise Radiation for Ground-Based Noise Measurements,” Master’s Thesis, University of Maryland, College Park, MD, 2008. 